\title{
CARACTERIZACIÓN SOCIODEMOGRÁFICA DE FAMILIAS CON NIÑOS EN CONDICIÓN DE VULNERABILIDAD Y RIESGO DE ADQUIRIR INFECCIÓN RESPIRATORIA AGUDA
}

\section{SOCIODEMOGRAPHIC CHARACTERIZATION FAMILIES WITH CHILDREN IN A CONDITION OF VULNERABILITY AND RISK OF ACQUIRING ACUTE RESPIRATORY INFECTIONS}

\author{
Zorange Pungo Hidrobo, Alisson Dahiana Burbano Ospina, Honorio Medina Rodríguez, Edwin Mauricio \\ Millán Hernandez y Gustavo Adolfo Girón Restrepo \\ Semillero SIGES \\ Grupo de investigación en Salud Ambiente y Productividad-GISAP \\ Institución Universitaria Antonio José Camacho \\ Recibido: 31/03/2021 Aprobado: 11/05/2021 \\ Cómo citar este artículo: \\ Pungo Hidrobo, Z., Burbano Ospina, A.D., Medina Rodríguez, H., Millán Hernández, E.M. y Girón Restrepo, G.A.(2021). \\ Caracterización sociodemográfica de familias con niños en condición de vulnerabilidad y riesgo de adquirir infección \\ respiratoria aguda. Revista Sapientía, 13(25), 46 - 57.
}

\section{RESUMEN}

El presente trabajo de investigación tiene como objetivo general: la caracterización sociodemográfica y epidemiológica de familias vulnerables con niños menores de 5 años en riesgo de adquirir Infección Respiratoria Aguda, de las cuales se desprenden los siguientes objetivos específicos: establecer criterios para la selección de la población objeto de estudio; describir las condiciones sociodemográficas y epidemiológicas presentes en la población objeto de estudio y clasificar los determinantes sociales de salud involucrados en el riesgo de desarrollo de las Infecciones Respiratorias Agudas en menores de 5 años. Se realizó un estudio con alcance descriptivo, diseño transversal y de enfoque cuantitativo, donde se utilizó como técnica e instrumento de recolección de datos la encuesta (diseñada por el equipo de trabajo), aplicada al cuidador. Se llegó a las siguientes conclusiones: la población de niños menores de 5 años de edad que pertenecen al barrio Zamorano, comuna vulnerable de Palmira, se encuentran en un $40 \%$ de riesgo de padecer infecciones respiratorias agudas en lo que influye el nivel socioeconómico al que pertenecen; igualmente, se observó que el 60\% se encuentra en riesgo epidemiológico de adquirir infecciones respiratorias agudas y se identificó que las infecciones respiratorias agudas en menores de cinco años continúa siendo un problema de salud en la ciudad de Palmira, barrio Zamorano.

\section{PALABRAS CLAVE}

Atención en salud, factores socioeconómicos, administración en salud, accesibilidad a los servicios de salud, prestación de atención en salud.

\section{ABSTRACT}

The present research work has as its general objective; the sociodemographic and epidemiological characterization in vulnerable families with children under 5 years old at risk of acquiring Acute Respiratory Infection, from which the following specific objectives emerge: Establish criteria for the selection of the population under study. Describe the sociodemographic and epidemiological conditions present in the population under study. To classify the social determinants of health involved in the risk of development of Acute Respiratory Infections in children less than 5 years of age. A study was carried out with descriptive scope, 
cross-sectional design and mixed approach, where the survey (designed by the work team) applied to the caregiver was used as a technique and data collection instrument, reaching the following conclusions: The population of young children of 5 years of age that belong to the neighborhood Zamorano vulnerable commune of Palmira, is in $40 \%$ of suffering from acute respiratory infection where influences the socioeconomic level to which they belong, It was also observed that $60 \%$ is at epidemiological risk of acquire acute respiratory infections and it was identified that acute respiratory infections in children under five years of age continues to be a health problem in the city of Palmira Zamorano neighborhood.

\section{KEYWORDS}

Public Health, socioeconomic factors, health policy, health services accessibility, delivery of health care.

\section{INTRODUCCIÓN}

Como explica Teper et al (1999), la Infección Respiratoria Aguda (IRA) incluye un conjunto de enfermedades que afectan el sistema respiratorio, pueden ser causadas por microorganismos virales o bacterianos, representan una de las primeras causas de atención médica en todo el mundo, tanto en consulta externa como en hospitalización y se encuentran entre las primeras causas de mortalidad. Estas enfermedades afectan a toda la población, pero, fundamentalmente, a menores de cinco años. Según el Instituto Nacional de Salud de Colombia, en el mundo la Infección Respiratoria Aguda (IRA) representa dos millones de muertes cada año (Malo y Pulido, 2017). Asimismo, Hidalgo (1990) indica que, como promedio, un niño residente en un área urbana sufre entre 5 y 8 episodios de infecciones respiratorias agudas al año, con una duración media de 7 a 9 días, la mayoría de ellos se debe a infecciones menos graves de vías respiratorias superiores de origen viral.

De acuerdo con los datos estadísticos, a nivel regional del Departamento del Valle del Cauca (2010-2011), las infecciones respiratorias agudas causan la muerte a 42 mujeres menores de 1 año por cada 100.000 habitantes y en hombres menores de 1 año causan la muerte de 35 por cada 100.000 habitantes. Otra variable fundamental de la investigación es el concepto de vulnerabilidad social, que tiene dos componentes explicativos, según plantea Pizarro (2001):

Por una parte, la inseguridad e indefensión que experimentan las comunidades, familias e individuos en sus condiciones de vida a consecuencia del impacto provocado por algún tipo de evento económico social de carácter traumático. Por otra parte, el manejo de recursos y las estrategias que utilizan las comunidades, familias y personas para enfrentar los efectos de ese evento.

Este concepto es el que nos servirá para entender la problemática desde una visión estructurada acorde con las condiciones propias de nuestros países latinoamericanos en vías de desarrollo.

\section{MARCO TEÓRICO}

La Organización Mundial de Salud (OMS ) y el Fondo de las Naciones Unidas para la Infancia-UNICEF en 1992 proponen una estrategia enfocada en la atención integral de los niños y niñas menores de cinco años; estrategia que lleva por nombre Atención Integrada a las Enfermedades Prevalentes de la Infancia (AIEPI), implementada en Colombia en el año 1999, con el fin de brindar mayor equidad en la atención en salud de gestantes, niñas y niños y así mitigar las altas tasas de mortalidad, muertes que pudieron haber sido evitadas si estas enfermedades infecciosas hubieran sido atendidas oportunamente. Las enfermedades infecciosas en nuestro país afectan de forma inevitable a la primera infancia $y$, a pesar de que se ha buscado mantener un equilibrio en el estado de niños y niñas, es necesario crear más acciones en cuanto a los determinantes que condicionan el bienestar de la población en general, como la provisión de agua potable, nutrición y esquemas de vacunación completos (Gobernación del Norte de Santander, 2017). 
De acuerdo con el Ministerio de la Protección Social, el total de la población afiliada al Sistema General de Seguridad Social en Salud (SGSSS) debe ser un foco de análisis de riesgos y circunstancias presentadas, ya sean de forma individual o colectiva, que les permita identificar y priorizar los riesgos que se puedan presentar dentro del territorio y, de esta manera, prevenir y aminorar estos riesgos mediante acciones de promoción y prevención a cargo de las Entidades Promotoras de Salud (EPS) y las Entidades Administradoras de Planes de Beneficio (EAPB); son estas las entidades encargadas de la caracterización poblacional por cada departamento, municipio o distrito donde hayan afiliados. Para esto, la Dirección de Epidemiología y Demografía elaboró una guía conceptual y metodológica, con el fin de promover, orientar y dirigir la situación de salud, la cual está compuesta por cuatro capítulos (Minsalud, 2017).

El principal objetivo de la caracterización es obtener información completa de las condiciones sociales, económicas, psicológicas, culturales y políticas de la población en estado de vulnerabilidad en nuestro país, con el fin de ajustar las necesidades y encontrar posibles soluciones. La Real Academia de la Lengua Española, en una de sus definiciones, señala que caracterizar es "determinar los atributos peculiares de alguien o de algo, de modo que claramente se distinga de los demás" (RAE, 2017). De acuerdo con esto, identificamos que caracterización es determinar las particularidades de una persona, animal o cosa y que además lo diferencian del resto de su especie.

Según la OMS (2008), las condiciones de desigualdad en que las personas viven, crecen, trabajan y envejecen influye mucho en materia de salud, y lo más injusto es que la mayoría de las desigualdades son evitables, por ende, la acción sobre los determinantes de la salud fortalece a las personas, las comunidades y los países (OMS, 2008). De acuerdo con esto, existen determinantes intermedios y estructurales que se encuentran íntimamente relacionados con la salud. Estos determinantes hacen referencia a las condiciones que pueden ser modificadas, condiciones de vida, de vivienda, de trabajo, entre otros, los hábitos y los estilos de vida como particularidad que puede ejercer un impacto positivo o negativo en la salud (OMS, 2008).

El Ministerio de la Salud y la Protección Social (2011) define la vulnerabilidad como el conjunto de características que limitan la capacidad de los individuos. De igual forma, la Federación Internacional de Sociedades de la Cruz Roja y de la Media Luna Roja explica que esta puede definirse como:

la capacidad disminuida de una persona o un grupo de personas para anticiparse, hacer frente y resistir a los efectos de un peligro natural o causado por la actividad humana, y para recuperarse de los mismos, casi siempre se asocia con la pobreza, pero también son vulnerables las personas que viven en aislamiento, inseguridad e indefensión ante riesgos, traumas o presiones. (IFRC, 2021)

La vulnerabilidad tiene su origen en la interacción de factores internos, como son los recursos y estrategias de acción de los que disponen los individuos y los factores externos a los hogares. En este sentido, la vulnerabilidad de un individuo u hogar depende de la forma en que contrarreste los factores externos con los internos y su poder de resiliencia. Celidoni (2011) especifica la existencia de tres definiciones: vulnerabilidad como pobreza esperada (VEP) es la probabilidad de caer en un estado de pobreza en el futuro; vulnerabilidad como baja utilidad esperada (VEU) es la brecha entre el nivel de consumo intertemporal y el correspondiente a la pobreza vista como utilidad y, por último, la vulnerabilidad como exposición a riesgos no asegurables (VER) se enfoca en la relación entre consumo e ingresos del hogar.

Sánchez \& Jiménez (2011) plantean que el enfoque de la vulnerabilidad social se compone de vulnerabilidad demográfica y vulnerabilidad sociodemográfica; en ambos casos, las variables demográficas permiten identificar grupos vulnerables y riesgos sociodemográficos. Con base en la (CEPAL, 2001), la vulnerabilidad demográfica está constituida por las debilidades, desventajas o riesgos que tiene la 
comunidad en general como consecuencia de la intervención de factores de origen demográfico como tendencias o conductas; por su parte, Rodríguez (2000) señala que las variables sociodemográficas pueden actuar de forma negativa en el desarrollo tanto personal como familiar (Sánchez \& Jiménez, 2011).

Según la OMS (2007), las Enfermedades Respiratorias Agudas son un conjunto de enfermedades infecciosas que afectan las vías respiratorias, causadas por bacterias o virus que ingresan por boca o nariz. La Dirección General de Sanidad Militar-DGSM (2015) expresa que entre las ERA más frecuentes se encuentra el Resfriado Común, la Influenza, el Asma y la Neumonía; estas enfermedades se presentan en las personas de cualquier género y cualquier edad, siendo más vulnerables el adulto mayor y los niños en su primera infancia.

Según Valencia (2017), en Colombia, durante el periodo de agosto a diciembre de 2014, fueron evaluados diferentes diagnósticos clínicos como neumonía, asma y rinofaringitis, donde se pudo verificar que de 394 niños menores de 5 años que asistieron al servicio hospitalario, 105 presentaron un diagnóstico de ERA, esto es el 26,7\% de los casos, siendo las Infecciones Respiratorias Agudas (IRA) las de mayor incidencia, correspondientes al 7,7\% de las consultas externas y urgencias atendidas y al 7,4\% de las hospitalizaciones.

\section{METODOLOGÍA}

Alcance: el presente estudio fue de alcance descriptivo analítico, con un diseño transversal ejecutado en 12 meses (junio de 2016 a junio de 2017), donde se incluyó trabajo de campo y revisión de estadísticas que corroboran datos epidemiológicos entre los años 2012 y 2016.

Enfoque: para el desarrollo de la investigación se usó un enfoque cuantitativo.

Población: se siguió la siguiente ruta metodológica. Se buscó la información correspondiente en la ciudad de Palmira (Colombia), donde se encontró que la Alcaldía tiene plenamente identificada la comuna 1 y 2 como las más vulnerables socioeconómicamente de la ciudad. Con datos de la Secretaría Municipal de Salud de Palmira se realizó el siguiente paso, basado en cruzar criterios de información de incidencia de Infecciones Respiratorias Agudas-IRA, en niños menores de 5 años, en un periodo de 5 años, con este paso se identificó que en la comuna 1 con presencia de población vulnerable tiene un número mayor de casos de IRA. La selección de las viviendas se realizó por conveniencia.

Muestra: la técnica utilizada fue el modelo Ad Hoc no probabilístico, este fue utilizado por los problemas de orden público que presenta la zona. En Palmira, la comuna 1 tiene un total de 38.723 habitantes, organizados en 21 barrios, sin embargo, el barrio Zamorano tiene un $36 \%$ de la población total de la comuna, con un total de 13.933 habitantes, ninguno de los otros 20 barrios llega al 11\% de la población. Por tal motivo, se tomó como referencia el barrio Zamorano, se tomaron datos que dicen que viven 4 personas por hogar, según el censo del Departamento Nacional de Estadística de Colombia para la zona, por lo cual se estiman 3.483 hogares en total, después se usó la fórmula estadística para calcular el tamaño de muestra llegando a una muestra representativa de 240 hogares en el barrio Zamorano de Palmira.

\section{ESTRUCTURA DEL ESTUDIO}

Fase 1. Conocer y desarrollar el proyecto de investigación: se dieron a conocer objetivo y finalidad del estudio. A partir de este aspecto se revisó el estado del arte que permitió conocer el tema a desarrollar.

Fase 2. Definición de variables para realizar el instrumento: se realizó una agrupación de diferentes opciones de variables que estuvieran alineadas con los objetivos de la investigación y que permitieran por medio de un instrumento (encuesta) dar respuesta a la investigación.

Fase 3. Construcción del Instrumento: en la creación 
del instrumento se abordaron aspectos como: identificación del cuidador de los niños, condiciones de la vivienda, condiciones del medio ambiente, promoción y prevención, identificación estado de salud del menor, condiciones de salud y hábitos de vida cotidiana. La encuesta está conformada por 4 páginas y 7 ítems donde cada uno tiene una breve explicación de lo que se quiere preguntar; el primer bloque corresponde a la identificación e información del cuidador principal, consta de 15 preguntas cerradas y 2 abiertas; el segundo bloque a las condiciones de la vivienda y población objeto, consta de 7 preguntas cerradas; el tercer bloque corresponde a la condiciones de medio ambiente, consta de 5 preguntas cerradas y una abierta; el cuarto bloque corresponde a la promoción y prevención, consta de 4 preguntas cerradas; el quinto bloque es sobre identificación del estado de salud del menor, consta de 6 preguntas abiertas y 9 preguntas cerradas; el sexto bloque corresponde a las condiciones de salud y hábitos de vida cotidiana en la familia, consta de 8 preguntas cerradas y el séptimo bloque corresponde a las rutas de atención integral en salud, consta de 7 preguntas cerradas y 1 abierta.

Fase 4. Prueba piloto: se realizó una prueba piloto con 20 encuestas en la comuna 1 de Palmira, en otro barrio diferente al Zamorano, pero con condiciones similares; se identificaron aspectos como: tiempo de aplicación de la encuesta en una familia, contacto con los líderes comunitarios de la zona, comprensión de las preguntas por los entrevistados, de esa forma se logró el diseño definitivo del instrumento.

Fase 5. Aplicación del instrumento: se aplicó el instrumento a 240 hogares del barrio Zamorano del municipio de Palmira durante 30 días, con niños menores de 5 años en riesgo de padecer Infección Respiratoria Aguda.

Fase 6. Sistematización y análisis de los resultados: se recolectó la información y se procedió a procesar su digitación manual en un aplicativo en Excel que permitió entregar datos y gráficos para el posterior análisis.
Consideraciones éticas: el estudio fue aprobado por el comité de ética hospitalaria de la Red de Salud de Ladera E.S.E mediante acta de aprobación No. 01-CEH/0202-17 B/N-01. En esta investigación predominaron los criterios de respeto a la dignidad humana, a la protección de los derechos y el bienestar de los participantes, protegiendo siempre la privacidad del individuo, identificándolo solo cuando los resultados lo requieran y este lo autorice, de acuerdo con la Resolución № 008430 de 1993 del Ministerio de la Protección Social que establece las normas científicas, técnicas y administrativas para la investigación en salud. El consentimiento informado se presentó verbalmente $\mathrm{y}$, a través de este, los participantes conocieron el objetivo y propósito de la investigación.

Durante la investigación se respetó la persona como a un ser holístico, autónomo, único, libre e independiente que tiene el derecho y la capacidad de tomar su propia decisión de participar o no en la investigación; previa información verbal brindada de manera clara, sencilla y necesaria se procedió a realizar la encuesta.

Se les aseguró que los datos obtenidos no serían utilizados en beneficio propio ni de ninguna otra institución, por ello se cuidó la integridad moral, psicológica, social y espiritual de la población en estudio. Durante el desarrollo del estudio se cumplieron los siguientes principios éticos de investigación. El principio de justicia, cada participante recibió un trato justo, digno; asimismo, se utilizaron los datos solo con fines de la investigación, con estricta privacidad, es decir, se respetó el derecho de anonimato.

\section{HALLAZGOS Y RESULTADOS}

Según datos de las encuestas realizadas, en el $80.83 \%$ de los hogares vive un niño, en el $15.83 \%$ viven dos niños, en $2.5 \%$ viven tres niños y en el $0.83 \%$ viven cuatro niños; en cuanto a las edades de los cuidadores de los niños, 70\% tiene edades entre 18 y 40 años, entre 41 y 
50 años el 14.58\% y entre 51 y 60 el 15\%; con relación al género del cuidador un $0.8 \%$ masculino y el $99.2 \%$ femenino. Con respecto al estado civil de los cuidadores, un 57,08\% vive en unión libre, el 14.17\% son separados, el $11.25 \%$ es casado, el $9.17 \%$ en viudo y el $8.33 \%$ es soltero. En cuanto a la conformación de la familia, de las 240 familias encuestadas el 32.5\% están conformadas por una familia, el $30.42 \%$ por dos familias, el $12.92 \%$ por tres familias, el $12.1 \%$ por cuatro familias, el $3.8 \%$ por cinco familias, el $2.5 \%$ por seis familias y el 5.8\% más de 6 familias; las familias están conformadas en un $64.17 \%$ por papá, mamá, hijos y abuelos, un $35 \%$ por papá, mamá e hijos y un $0.83 \%$ mamá e hijos. Al analizar el nivel educativo de los encuestados se encontró que el $4.2 \%$ de los cuidadores no tienen estudios o no han cursado algún tipo de enseñanza, y el 70,4\% no términó la primaria, lo que nos muestra un claro problema de orden educativo. De las personas encuestadas el $48.75 \%$ son amas de casa, el $26.3 \%$ es desempleado, el $8.33 \%$ es empleado, el $7.08 \%$ es independiente, el $5.42 \%$ es pensionado y el $4.17 \%$ estudia.

Con relación al ingreso mensual de las familias un $59.6 \%$ tiene salario mínimo, más de un salario mínimo $37.5 \%$ y menos de un salario mínimo el 2.9\%, según los hallazgos de la muestra.

En relación con la tenencia de la vivienda $47.1 \%$ viven la casa alquilada, el $37.9 \%$ viven en casa familiar, el $14.6 \%$ viven en casa propia y el $0.4 \%$ la casa es prestada. $\mathrm{Al}$ analizar se observa que el $99.6 \%$ de las familias encuestadas vive en casa hecha de material de ladrillo y el $0.4 \%$ de bareque; el $51.7 \%$ tienen piso en cerámica y el $48.3 \%$ cuenta con piso de cemento. En cuanto a las condiciones de la vivienda, se observó que en el $40.8 \%$ el techo de las viviendas es plancha, el $29.2 \%$ es de Eternit, el 25\% en tejas y el 5\% zinc. Con relación a las condiciones del medio ambiente, el $100 \%$ de las familias encuestadas cuentan con servicio de acueducto, el 100\% de las familias encuestadas cuentan con servicio de energía eléctrica, el 100\% de las familias encuestadas cuentan con servicio de recolección de basuras, se evidenció que el $55.8 \%$ usa gas natural y el $37.5 \%$ usa gas de pipa, solo un $4 \%$ usa leña. En el total de las casas encuestadas el $80.4 \%$ vive con mascotas y el $19.6 \%$ no viven con mascotas.

De las personas encuestadas, el $84.2 \%$ sí conoce el centro de salud al cual puede dirigirse y el $15.8 \%$ no tiene conocimiento; del total de los niños encuestados, el 99\% tiene esquema de vacunación y el 1\% no tienen; con respecto a la lactancia materna, el $79 \%$ de los menores recibió lactancia materna y el $21 \%$ no recibió. Un dato importante es que el $40 \%$ de los menores ha padecido de Infecciones Respiratorias Agudas y el 60\% nunca lo han padecido.

\begin{tabular}{|c|c|c|}
\hline & Cantidad & Porcentaje \\
\hline Si & 117 & $40 \%$ \\
\hline No & 115 & $60 \%$ \\
\hline Total & 232 & $100 \%$ \\
\hline
\end{tabular}

Tabla 1. Padecimiento de Infección Respiratoria Aguda en la población en barrio Zamorano de Palmira (número de niños por vivienda) Fuente: Elaboración Propia

De los 117 niños que padecieron Infección Respiratoria Aguda, el 54\% ha sufrido de asma, el 36\% de bronquitis y el $10 \%$ de neumonía, destacándose el asma como el caso más frecuente en los niños menores de 5 años.

\begin{tabular}{|c|c|c|}
\hline Enfermedad & Cantidad & Porcentaje \\
\hline Asma & 63 & $54 \%$ \\
\hline Bronquitis & 42 & $36 \%$ \\
\hline Neumonia & 12 & $10 \%$ \\
\hline Total & 117 & $100 \%$ \\
\hline
\end{tabular}

Tabla 2. Enfermedades Respiratorias Agudas más se manifiesta en barrio Zamorano de Palmira Fuente: Elaboración Propia

Se observó que el $71.3 \%$ de los cuidadores no fuman y el $28.8 \%$ sí fuman, se evidenció que un $62.1 \%$ del grupo familiar de las personas encuestadas sí fuman y el 37.9\% no fuman; en las familias se encontró que el $64.2 \%$ no ha padecido de enfermedad respiratoria, el $22.5 \%$ han padecido de asma, el $7.9 \%$ de neumonía, el $4.6 \%$ de bronquitis, el $0.4 \%$ de Enfermedad Pulmonar Obstructiva Crónica (EPOC) y el 0.4\% de tuberculosis. 


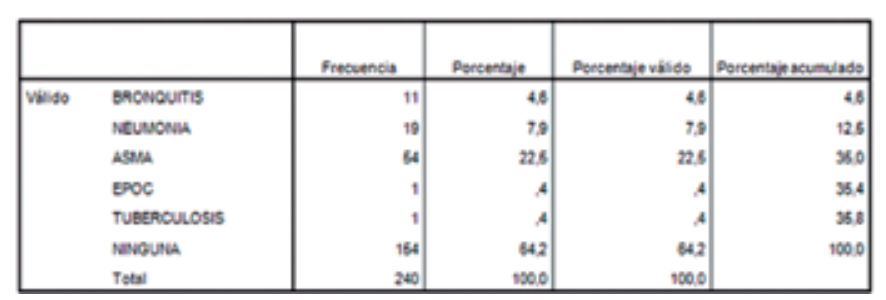

Tabla 3. Dentro del grupo familiar alguno ha padecido de Enfermedad Respiratoria Aguda en barrio Zamorano de Palmira Fuente: Elaboración Propia

- $\quad$ ¿Tipo de régimen de seguridad social en salud? El $67.9 \%$ pertenece al Régimen Subsidiado (población pobre asegurada), el 25.8\% al Régimen Contributivo (población con capacidad de pago) y el $6.3 \%$ (población pobre no afiliada) PP-NA.

- ¿ ¿Conoce el centro para la atención integral de salud del infante? De las personas encuestadas, el $84.2 \%$ sí conoce el centro de salud al cual puede dirigirse y el $15.8 \%$ no tiene conocimiento.

- Las personas que respondieron afirmativamente que conocen el centro de salud: se observó que el $62.92 \%$ no tienen conocimiento cómo se llama, el $20.42 \%$ van al hospital, el $8.33 \%$ a Coomeva, el $5 \%$ a Comfandi, el $2.08 \%$ a Comfenalco, el $0.42 \%$ a Clínica Palmira y el $0.83 \%$ a Salud Total.

- ¿El desplazamiento de su casa al centro de salud es seguro? El 67.1\% respondieron que se sienten seguros desplazándose al centro de salud, un $32.92 \%$ aseguraron que no es seguro

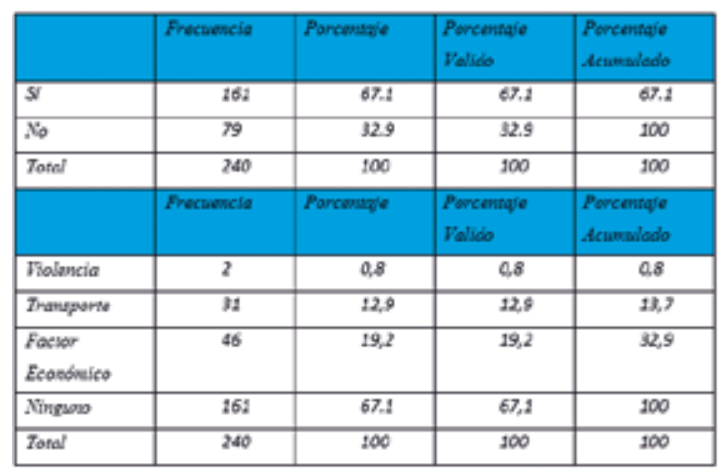

Tabla 4. El desplazamiento de su casa al centro de salud es seguro. ¿Por qué no es seguro el desplazamiento al centro de salud? Fuente: Elaboración Propia
- De las personas que respondieron que el desplazamiento de su casa al centro de salud no es seguro: el $19.2 \%$ dice que es por factor económico, el $12.9 \%$ por falta de transporte, el $0.8 \%$ por violencia y el $67.08 \%$ no aplica.

- ¿ ¿Por qué medio solicita la cita de control y desarrollo para el niño/niña? El medio por el cual solicitan las citas de control y desarrollo es con un $64.17 \%$ personalmente, un $35 \%$ vía telefónica y el $0.83 \%$ por Internet.

\section{DISCUSIÓN}

En el aspecto sociodemográfico, la edad del cuidador se encuentra entre los 18 y 40 años, son en su mayoría mujeres, se dedican al hogar (amas de casa) y solo pudieron acceder a la educación básica, los padres de familia viven en unión libre y el ingreso familiar es de un salario mínimo mensual legal vigente; en cada casa habitan de 1 a 2 familias promedio, conformadas en su mayoría por mamá, papá, hijos y abuelos. Estos resultados guardan relación con los de Alonso Cordero (2008), quien señala una asociación estadística significativa entre factores sociales, escolaridad materna e Infección Respiratoria Aguda y plantea que el analfabetismo es un factor de riesgo para tener en cuenta en la incidencia de Infección Respiratoria Aguda. De manera que el bajo nivel escolar, cultural y económico son elementos de gran consideración.

En lo que se refiere al género de los menores se halló que predominó el masculino, siendo en su mayoría niños de 3 años, en general se encontró que el peso al nacer fue de 2920 a 3300 gr, el cual está dentro de un rango normal; esto es importante ya que el bajo peso puede conllevar a una alimentación inadecuada $y$, por lo tanto, ser mucho más propensos a enfermedades. Niños de 3 meses y 24 meses tienen un porcentaje considerable de bajo peso según la muestra, notando una desnutrición que puede llevar a déficit en el crecimiento y desarrollo. En el estudio de Alonso 
Cordero (2008), los resultados fueron que el bajo peso al nacer no es un factor de riesgo a considerar para la aparición de las Infección Respiratoria Aguda, ya que solo hubo 7 niños con bajo peso del total de la muestra, $8,8 \%$.

Casi en su totalidad, los menores cuentan con carnet de vacunación, los padres son conscientes de la importancia de tenerlo, pero resaltando que muchos no tienen el esquema completo, el cual es importante porque las vacunas constituyen una de las medidas sanitarias que mayor beneficio ha producido en la prevención de enfermedades.

Por otro lado, se obtuvo que más de la mitad de los menores recibió lactancia materna por más de 6 meses, siendo importante en los niños para reducir significativamente la Infección Respiratoria Aguda y otras enfermedades. La lactancia materna mejora el estado inmunológico de los infantes. Los bebés alimentados a pecho presentan un mejor estado nutricional en los primeros meses de vida, lo que puede contribuir a la reducción en la incidencia y gravedad de las enfermedades infecciosas. Aquellos niños que al nacer no reciben lactancia materna, no tienen la protección necesaria contra la infección respiratoria aguda, por lo tanto, la incidencia será mayor. Estos resultados se asemejan a los encontrados en el estudio de factores de riesgo para infección respiratoria aguda realizado por Corredor Gamba et al. (2015) en los barrios Ciudad Jardín y Pinos de Oriente de Tunja, Colombia, donde entre los factores de riesgo se consideró la lactancia materna; el 93\% de los menores recibieron lactancia materna, de los cuales el 35\% la recibieron de 7 a 12 meses, el 24\% hasta los 6 meses y el $24 \%$ por más de 19 meses.

La investigación estuvo representada por 232 menores, en la cual el $40 \%$ han padecido de Enfermedad Respiratoria Aguda y en mayor concentración han padecido de asma (54\%). Estos resultados contrastan con el estudio de Fernández Cardona (2009), donde el $16 \%$ de los niños tendrían un alto riesgo de asma y existe un factor de riesgo hereditario positivo en la primera línea de consanguinidad, que va de un $11 \%$ para asma hasta un $25 \%$ para rinitis. hereditario positivo en la primera línea de consanguinidad, que va de un $11 \%$ para asma hasta un $25 \%$ para rinitis.

En lo que se refiere al hábito de fumar en los cuidadores de los niños, 171 personas de los 240 hogares respondieron que No fumaban; es un buen porcentaje ya que los cuidadores son los que están más tiempo con ellos, sin embargo, cabe aclarar que el hábito de fumar en los convivientes se presentó en 149 personas, representando el 62,08\%, convirtiendo los niños en fumadores pasivos. Según el estudio realizado por (Rabines, 2002) el humo del cigarrillo contiene sustancias tóxicas que afectan el mecanismo de depuración mucociliar y la actividad de macrófagos, aumentando la producción de mucus y favoreciendo la aparición de las Infección Respiratoria Aguda. El estudio realizado por Jaimes et al (2003) demuestra la asociación entre el humo ambiental del tabaco en fumadores pasivos y las enfermedades respiratorias en la niñez. Los hijos de los fumadores no presentan tan buenos resultados en las pruebas de función pulmonar y muestran de 1,5 a 2,0 veces mayor incidencia de infecciones respiratorias bajas que los hijos de los no fumadores. En el presente estudio fumar dentro de la vivienda aumentó en $43 \%$ el riesgo de Infección Respiratoria Aguda grave en los niños, comparado con los niños en cuya vivienda no había fumadores.

En el estudio de Rodríguez (2010) en cuanto a los determinantes de la salud y los factores biológicos que involucra el padecimiento de enfermedad respiratoria más la ruta de riesgo, los antecedentes de los familiares aumentan las posibilidades de que el niño sufra de Infección Respiratoria y asma, además este riesgo aumenta cuando en la casa hay familiares que fuman en presencia del niño que tiene problemas respiratorios. Es decir, el riesgo de asma está definido en función del antecedente familiar y la exposición a tabaco en un niño que responde a ruidos.

Los datos arrojados por la encuesta muestran que las viviendas estaban ubicadas en un barrio vulnerable que se caracterizaba por tener techos de plancha (40.8\%), pisos de cerámica $(51.7 \%)$ y otro piso en cemento 
(48.3\%). Los animales domésticos (perros, gatos y aves) fueron factores ambientales con mucha frecuencia en las viviendas encuestadas (80.4\%).

Según Herrera (2000), las condiciones de la vivienda donde los niños pasan gran parte de su vida, pueden ser determinantes para la presencia de infección respiratoria aguda; es así como los problemas de infraestructura, hacinamiento, almacenamiento y recolección de basuras, poca ventilación, contaminación dentro de esta, piso en tierra, calles de acceso sin pavimentar y convivencia con animales tendrán impacto en la salud de los niños.

Continuando con lo encontrado en el estudio de Herrera (2000), los resultados muestran que el hacinamiento se presenta con mayor frecuencia en la primera habitación donde duermen 3 personas, por lo que se comportó como un factor de riesgo. Los niños que duermen en habitaciones donde hay más de tres personas tienen mayor predisposición a adquirir IRA, pues los adultos portan en las vías respiratorias microorganismos que se mantienen de forma asintomática, pero que son transmitidos por medio de la tos, el estornudo o el contacto directo.

El comportamiento de la cobertura del Sistema General de Seguridad Social en Salud de las familias encuestadas evidenció que 163 personas pertenecen al régimen subsidiado, el cual garantiza los servicios de salud de la comuna, teniendo atención médica y hospitalaria. De acuerdo a datos del Boletín de aseguramiento No 01 del primer trimestre 2017 sobre el número de afiliados al Sistema General de Seguridad Social en Salud, el 97.3\% de los colombianos está plenamente cubierto, $44.5 \%$ hacen parte del régimen contributivo (población con capacidad de pago), $48.1 \%$ hacen parte del régimen subsidiado (población pobre asegurada) y $5 \%$ hacen parte de los regímenes especiales o de excepción (Ministerio de Salud, 2017).

El desplazamiento de la casa al centro de salud en un gran porcentaje respondió que es seguro, sin embargo, los que respondieron que no, esto se debe al factor económico. En el estudio sobre la prevalencia de barreras de acceso a los servicios en salud en San GilSantander, realizado por Agudelo Estupiñán (2013), la principal barrera de acceso a los servicios de salud fue la oportunidad en la atención con un $81.26 \%$, dentro de ellas la de mayor frecuencia fue la solicitud de la consulta de medicina general mayor a 3 días. La siguiente barrera de acceso con mayor relevancia fueron las dificultades en la atención con $29.32 \%$, de ellas la de mayor frecuencia fue la tramitología con 86.4\%. Las limitaciones para cumplir una cita por falta de recursos económicos también tuvieron una importante manifestación con un $11.52 \%$.

\section{CONCLUSIONES}

La población de niños menores de 5 años de edad que pertenecen al barrio Zamorano, comuna vulnerable de Palmira, se encuentra en un $40 \%$ de padecimiento de infección respiratoria aguda, situación en la que influye el nivel socioeconómico al que pertenecen y factores como nivel de educación, ingreso familiar, características de vivienda, frecuencia de ingresos y ocupación laboral.

Igualmente, se observó que el $60 \%$ se encuentra en riesgo epidemiológico de adquirir infecciones respiratorias agudas, se tuvieron en cuenta factores como: peso al nacer y actual, talla al nacer y actual, esquema de vacunación, lactancia materna, padecimiento de enfermedad respiratoria, factores a los cuales se les debe realizar seguimiento y control. En esta investigación se identificó que las infecciones respiratorias agudas en menores de cinco años continúan siendo un problema de salud en la ciudad de Palmira, barrio Zamorano, localidad donde, sin embargo, cuentan con un puesto de salud que cubre sus necesidades de manera eficiente; su población en gran mayoría pertenece al régimen subsidiado, la población encuestada tiene conocimiento de la ubicación del puesto de salud, aunque se asegura que por falta de recursos económicos no se puede desplazar hasta él.

\section{AGRADECIMIENTOS}

Agradecimientos al Grupo de Investigación en Salud, 
Ambiente y Productividad (GISAP), al Semillero de Investigación de Gestión en Salud SIGES del programa Administración en Salud, a la Institución Universitaria Antonio José Camacho, al Decanato Asociado de Investigaciones y a la decanatura de la Facultad de Educación a Distancia y Virtual.

\section{REFERENCIAS BIBLIOGRÁFICAS}

Agudelo Estupinán, A. C., Gómez Amaya, P., Montes González, J. y Pelayo, G.B. (2013).

Barreras de acceso a los servicios de salud en el régimen subsidiado del Municipio de San Gil-Santander (trabajo de grado). Universidad CES, Santander, Colombia.

Alonso Cordero, M., Rodríguez González, N., Rodríguez Carrasco, B., \& Hernández Gómez, L. (2008). Infecciones respiratorias agudas en niños menores de 5 años. Primera parte. Medimay, 14(2), 46-56. http://revcmhabana. sld.cu/index.php/rcmh/article/view/297/520

CEPAL. (2001). Panorama Social de América Latina 2000-2001. Organización de Naciones Unidas. Obtenido de https://www.cepal.org/es/ publicaciones/1211-panorama-social-americ a-latina-2000-2001

Celidoni, M. (2011). Vulnerability to poverty: An empirical comparison of alternative measures. https://mpra.ub.uni-muenchen. $\mathrm{de} / 33002 /$

Corredor Gamba, S., Umbacia Salas, F., Sandoval-Cuellar, C., \& Rojas, P. (2015). Factores de riesgo para infección respiratoria aguda en los barrios Ciudad Jardín y Pinos de Oriente, Tunja, Colombia. Revista Investigación en Salud Universidad de Boyacá, 2(1), 14-30. https://doi.org/10.24267/23897325.127
Dirección General de Sanidad Militar-DGSM., (2015). Enfermedad_Respiratoria_Aguda_ERA. Obtenido de Grupo Salud Pública: https://www. sanidadfuerzasmilitares.mil.co/comunicaciones/foto _noticias/enfermedad_respiratoria_aguda_eraFeder ación Internacional de Sociedades de la Cruz Roja-IFRC. (2021). ¿Qué es la vulnerabilidad? https://www.ifrc.org/es/introduccion/disaster-mana gement/sobre-desastres/que-es-un-desastre/que-es-l a-vulnerabilidad/

Fernández Cardona, A., Fontalvo Acosta, M.A., Gallego Valencia, T.A., Hinestroza Palomino, M.L., Pineda Gutiérrez, L.M., Reyes Arce, D.M., Martínez, J.W. \& Hernández, C.J. (2009). Frecuencia de alteraciones respiratorias en escolares de la florida en pereira. Investigaciones Andina, 11(18), 37-54. http://www.scielo.org.co/scielo.php?script=sci_artte xt\&pid=S0124-81462009000100004\&lng=en\&tlng= es.

Gobernación del Norte de Santander. (2017). Infancia del norte. https://infanciadelnorte. es.tl/Historia-de-AIEPI.htm

Hidalgo, E. A. (1990). Caracterización de las IRAs y factores de riesgo en niños menores de 5 años en la comunidad del Paraíso Región Sanitaria No. 1 (Tesis doctoral). Universidad Nacional de Honduras. Tegucigalpa, Honduras.

Jaimes, M.B., Cáceres, D.C., De la Hoz, F., Gutiérrez, C., Herrera, D., Pinilla, J., Porras, A., Rodríguez, F. \& Velandia, M. (2003). Factores de riesgo para infección respiratoria aguda baja grave en Bogotá, 2001. Biomédica, 23(3), 283-292. https://www.redalyc.org/articulo.oa?id=84323305.

Malo, D.M. y Pulido, P.A. (2017). Informe de evento Infección Respiratoria Aguda. Instituto Nacional de Salud. https://www.ins.gov. co/buscador-eventos/Informesdeevento/Infor me IRA Final 2017.pdf 
Ministerio de Salud y Protección Social. (2017). Guía conceptual y metodológica para la caracterización de la población afiliada a las Entidades Administradoras de Planes de Beneficios de Salud (EAPB). Ministerio de Salud y Protección Social. https://www.minsalud. gov.co/sites/rid/Lists/BibliotecaDigital/RIDE/V S/ED/GCFI/Guia_caracterizacion_EAPB\%20ve rsion\%2021072014.pdf

OMS. (2008). Enfermedades respiratorias agudas con tendencia epidémica y pandémica. World Health Organization.df

Pizarro, R. (2001). La vulnerabilidad social y sus desafíos: una mirada desde América Latina. Santiago de Chile: Naciones Unidas.

Prieto Herrera, M.E., Russ Durán, G. \& \& Reitor Landrian, L. (2000). Factores de riesgo de infecciones respiratorias agudas en menores de 5 años. Revista Cubana de Medicina General Integral, 16(2), 160-164. http://scielo.sld.cu/scielo. php?script=sci_arttext\&pid=S0864-212520000 00200010\&lng=es\&tlng=es.

Rabines, J. A. (2002). Factores de riesgo para el consumo de tabaco en población de adolescentes escolarizados. Peru. Obtenido de https://sisbib.unmsm.edu.pe/bibvirtualdata/tes is/salud/rabines_j_a/t_completo.pdf

Real Academia Española-RAE. (2017). Caracterizar. . https://dle.rae.es/caracterizar
Real Academia Española-RAE. (2017). Caracterizar. https://dle.rae.es/caracterizar

Rodríguez, L.A., Rey, J.J., Berena Herrera, A., Castro, H., Niederbacher, J., Vera, L.M., Libia Cala, L., \& Bolívar, F. (2010). Prevalencia de síntomas respiratorios indicativos de asma y asociación con contaminación atmosférica en preescolares de Bucaramanga, Colombia. Biomédica, 30(1), 15-22. https://www.redalyc. org/articulo.oa?id=84312378003

Sánchez-González, D. \& Egea-Jiménez, C. (2011). Enfoque de vulnerabilidad social para investigar las desventajas socioambientales. Su aplicación en el estudio de los adultos mayores. Papeles de Población, 17(69), 151-185. https://www.redalyc.org/articulo.oa?id=11221 117006

STeper, A. M., Kofman, C. D., Maffey, A. F., \& Vidaurreta, S. M. (1999). Lung function in infants with chronic pulmonary disease after severe adenoviral illness. The Journal of pediatrics, 134(6), 730-733. https://doi.org/10.1 016/s0022-3476(99)70289-1

Ministerio de Salud y Protección Social. (2017). Boletín 01 del aseguramiento en salud. Primer trimestre de 2017.

Zavala-González, M.A., García-Ulín, G., Jiménez-Sastré, A. y Quevedo-Tejero, E.C. (2010). Infecciones Respiratorias Agudas en preescolares del poblado Rubén Jaramillo Tecamachalco, Puebla. Revista médica UIS, 23(1), 35-42. 


\section{AUTORES}

Zorange Pungo Hidrobo: administradora en Salud, especialista en Desarrollo Humano y Organizacional, coordinadora de la zona 18 Red Salud Ladera E.S.E.

Alisson Dahiana Burbano Ospina: administradora en Salud.

Honorio Medina Rodríguez: administrador en Salud, especialista en Gestión Pública, promotor de programas sociales sucursal Valle de Coosalud.

Edwin Mauricio Millán Hernández: profesional en Salud Ocupacional, candidato a Doctor en Salud Pública, máster en Desarrollo Sustentable, profesor Investigador de la Institución Universitaria Antonio José Camacho. Correo: emmillan@admon.uniajc.edu.co

Gustavo Adolfo Girón Restrepo: odontólogo, máster en Salud Pública en proceso de grado, especialista en Gerencia en Servicios de Salud, profesor Investigador de la Institución universitaria Antonio José Camacho. Correo: gagiron@admon.uniajc.edu.co 